\title{
Tobacco seeds expressing feedback-insensitive cystathionine gamma-synthase exhibit elevated content of methionine and altered primary metabolic profile
}

Ifat Matityahu ${ }^{1+}$, Itamar Godo ${ }^{1+}$, Yael Hacham ${ }^{1}$ and Rachel Amir ${ }^{1,2^{*}}$

\begin{abstract}
Background: The essential sulfur-containing amino acid methionine plays a vital role in plant metabolism and human nutrition. In this study, we aimed to elucidate the regulatory role of the first committed enzyme in the methionine biosynthesis pathway, cystathionine $\gamma$-synthase (CGS), on methionine accumulation in tobacco seeds. We also studied the effect of this manipulation on the seed's metabolism.

Results: Two forms of Arabidopsis CGS (AtCGS) were expressed under the control of the seeds-specific promoter of legumin B4: feedback-sensitive F-AtCGS (LF seeds), and feedback-insensitive T-AtCGS (LT seeds). Unexpectedly, the soluble content of methionine was reduced significantly in both sets of transgenic seeds. Amino acids analysis and feeding experiments indicated that although the level of methionine was reduced, the flux through its synthesis had increased. As a result, the level of protein-incorporated methionine had increased significantly in LT seeds by up to $60 \%$, but this was not observed in LF seeds, whose methionine content is tightly regulated. This increase was accompanied by a higher content of other protein-incorporated amino acids, which led to $27 \%$ protein content in the seeds although this was statistically insignificantly. In addition, the levels of reducing sugars (representing starch) were slightly but significantly reduced, while that of oil was insignificantly reduced. To assess the impact of the high expression level of T-AtCGS in seeds on other primary metabolites, metabolic profiling using GC-MS was performed. This revealed significant alterations to the primary seed metabolism manifested by a significant increase in eight annotated metabolites (mostly sugars and their oxidized derivatives), while the levels of 12 other metabolites were reduced significantly in LT compared to wild-type seeds.
\end{abstract}

Conclusion: Expression of T-AtCGS leads to an increase in the level of total Met, higher contents of total amino acids, and significant changes in the levels of 20 annotated metabolites. The high level of oxidized metabolites, the two stress-associated amino acids, proline and serine, and low level of glutathione suggest oxidative stress that occurs during LT seed development. This study provides information on the metabolic consequence of increased CGS activity in seeds and how it affects the seed's nutritional quality.

Keywords: Amino acids, Cystathionine -synthase, Germination, Methinonine, Metabolism, Seeds, Storage proteins

\footnotetext{
*Correspondence: rachel@migal.org.il

'Equal contributors

'Laboratory of Plant Science, Migal Galilee Technology Center, P.O. Box 831,

Kiryat Shmona 12100, Israel

${ }^{2}$ Tel Hai College, Upper Galilee, Israel
} reproduction in any medium, provided the original work is properly cited. The Creative Commons Public Domain Dedication waiver (http://creativecommons.org/publicdomain/zero/1.0/) applies to the data made available in this article, unless otherwise stated. 


\section{Background}

The level of methionine (Met), the essential sulfurcontaining amino acid, limits the nutritional quality of crops. Met is also a fundamental metabolite in plants, since, in addition to its role as a protein constituent and its central role in the initiation of mRNA translation, it is also a precursor for the synthesis of essential metabolites through its first metabolite, $S$-adenosyl Met (SAM). Due to the nutritional and metabolic importance of Met, studies were performed to assess the factors that regulate its synthesis and accumulation in vegetative tissues (reviewed by [1-3]). However, only a limited number of studies have been performed to reveal the factors regulating its synthesis and accumulation in seeds (review by $[4,5]$ ).

Biochemical and genetic evidence show that Met is synthesized de novo in seeds through the aspartate family pathway (Figure 1) as it occurs in leaves. The first committed enzyme of the Met biosynthesis pathway, cystathionine $\gamma$-synthase (CGS), regulates the pathway by combining the carbon-amino skeleton (derived from aspartate) with the sulfur group (derived from cysteine) [3,6] (Figure 1). However, other studies performed in several plants such as Arabidopsis and wheat indicate that Met that was synthesized in vegetative tissues was converted to $S$-methyl Met (SMM) and then transported from these tissues to the developing seeds. In seeds, SMM is then converted back to Met by the activity of homocysteine $S$-methyltransferase (HMT) [7,8] (Figure 1).

While the debate about the role of SMM and aspartate pathways in Met synthesis in seeds is still ongoing, two recent studies reported that seed-specific expression of feedback-insensitive mutated forms of Arabidopsis CGS (AtCGS) in legume seeds $[9,10]$ lead to higher levels of Met. These experiments indicate that CGS plays a major role in controlling Met synthesis in legumes seeds. However, further studies are required to reveal the roles of CGS (in the aspartate family pathway) and HMT (in the SMM pathway) in seeds of other plants.

In the current study, we aimed at revealing the role of CGS in Met synthesis in tobacco (Nicotiana tabacum) seeds that belong to Solanaceae. Tobacco plants were selected for this study since we had used them previously as a model plant to study the regulatory role of Arabidopsis CGS (AtCGS) in Met accumulation in tobacco vegetative tissues [11-14]. Two forms of AtCGS were overexpressed in tobacco plants: full-length AtCGS (F-AtCGS) that is



Figure 1 The aspartate family of amino acids and biosynthesis pathways leading to methionine in seeds of higher plants. Key enzymes and metabolites are specified. Abbreviations: AK, aspartate kinase; HK, homoserine kinase; TS, threonine synthase; TDH, threonine dehydratase; CGS, cystathionine $\gamma$-synthase; CBL, cystathionine $\beta$-lyase; MS, methionine synthase; OAS, O-acetyl serine; OASTL, O-acetylserine(thiol)lyase; SAM, S-adenosylmethionine; SAMS, SAM synthase; MGL, methionine $\gamma$-lyase; SMM, S-methylmethionine; MMT, methionine S-methyltransferase; HMT, homocysteine methyltransferase; MTHF, methyl-tetra hydrofolate. 
feedback-sensitive to high levels of Met/SAM [15]; and truncated AtCGS (T-AtCGS) that is Met/SAM feedbackinsensitive [11]. While the level of soluble Met increased significantly in plants overexpressing the F-AtCGS by about two-fold compared to wild-type (WT), those overexpressing the T-AtCGS showed a 2.5 -fold increase in Met but significantly higher levels of Met that incorporated to proteins (about two-fold compared to those expressing the F-AtCGS) [11,12]. Tobacco plants were chosen for the study also since their capsule morphology enables us to apply Met or other metabolites to the developing capsules in order to assess the effect on amino acids and primary metabolites in the developing seeds [16].

In the current study, we aimed (i) to elucidate the role of CGS in Met synthesis in tobacco seeds; (ii) to study the role of the feedback inhibition of AtCGS on Met synthesis in seeds; and (iii) to study the metabolic consequences of enhanced CGS activity in these seeds.

The metabolic consequences of increased Met content have not yet been studied, and only a few studies have addressed the relationships between increased levels of other amino acids to seeds metabolism $[17,18]$. Developing seeds are an excellent system for studying developmentally controlled metabolic regulation because during seed development, there is a massive synthesis of fatty acids, sugars and amino acids, which are converted to their storage forms: proteins, starch and oil [19]. The results show that high expression level of T-AtCGS leads to higher total Met content and affect the level of 20 annotated metabolites. The results imply that oxidative stress might occur in the transgenic seeds expressing this form of CGS.

\section{Results and discussion}

The endogenous tobacco CGS is regulated by a high level of Met

In this study, we express in tobacco seeds the feedbacksensitive form of AtCGS (F-AtCGS) and its mutated form that is insensitive to Met (T-AtCGS). To distinguish between the effects of these two forms of AtCGS, it is important to know if the endogenous tobacco CGS (NtCGS) is down-regulated by a high content of Met similarly to AtCGS [15]. To determine this, tobacco seedlings (21 days old) were fed with $5 \mathrm{mM}$ Met, or with double distilled water (DDW) as a control, and the expression level of NtCGS was measured by quantitative real-time PCR (qRT-PCR). The results (Figure 2A, left panel) show that the level of NtCGS is sensitive to high levels of Met such as AtCGS and tomato CGS [20,21], but unlike potatoes [22] that belong to the Solanaceae family together with tobacco and tomato.

Most probably due to the major role of Met in plant metabolism, we expected that similar to Arabidopsis [38], NtCGS would express in all tissues of the tobacco plants. Thus, we assume that if a higher level of Met will be found

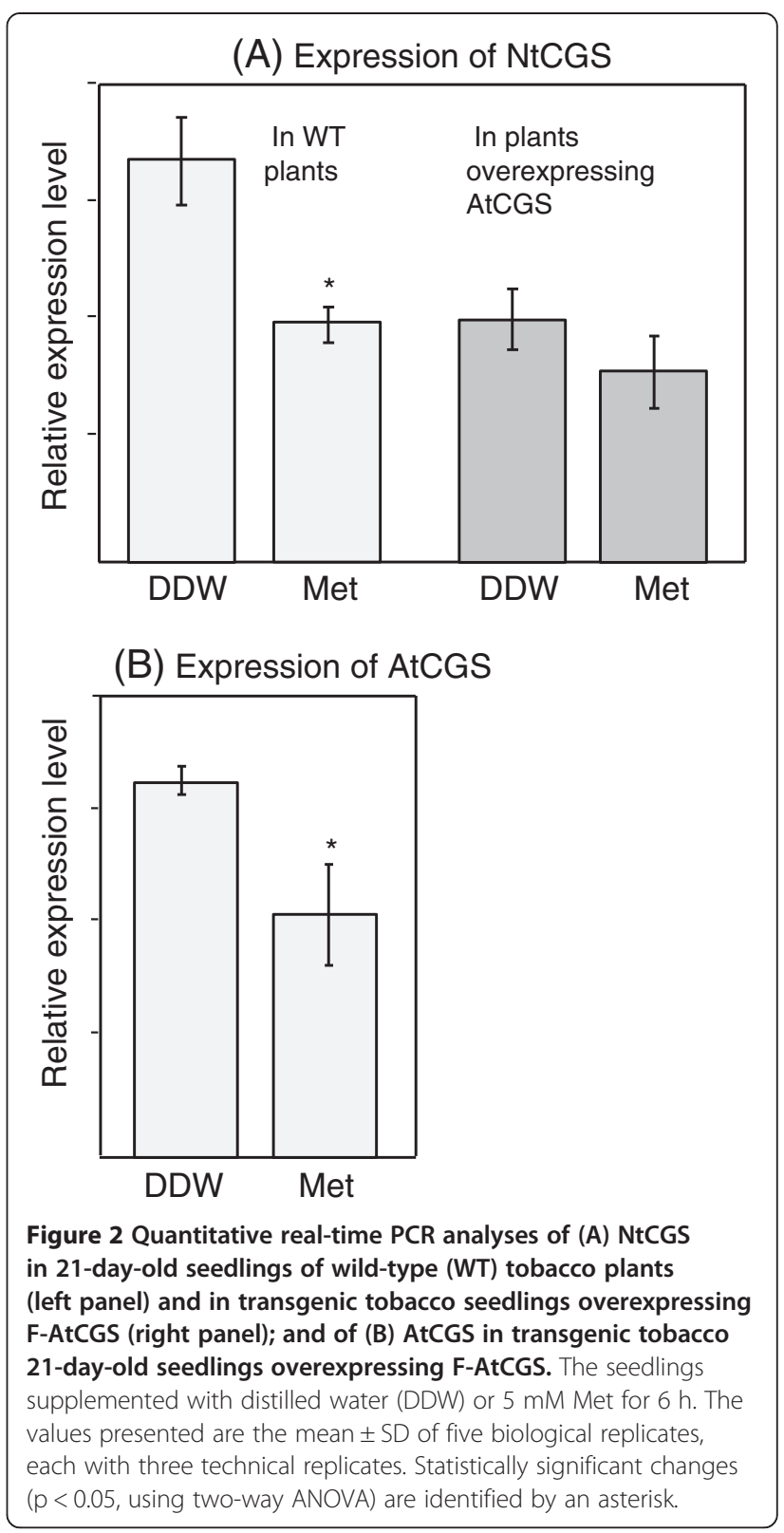

in the transgenic seeds expressing the AtCGS, it will be the result of the higher activity of AtCGS and less from the endogenous NtCGS.

\section{Seeds expressing AtCGS do not accumulate higher levels of soluble Met}

To assess whether CGS expression is a rate-limiting factor of Met synthesis in tobacco seeds, two forms of AtCGS (F-AtCGS; T-AtCGS) were expressed under the control of the seed-specific promoter, Legumin B4 [23], to produce LF and LT plants, respectively (Additional file 1: Figure S1). Seeds from 30 kanamycin-resistant plants from each line were screened by immunoblot to determine the expression level of the two forms of 
AtCGS (Additional file 1: Figure S2). Two plants showing the highest expression level for each line were selfpollinated to produce homozygous plants. Seeds from $\mathrm{T}_{3}$ lines were used for further analysis.

The levels of soluble Met were then measured in seeds of transgenic homozygous lines and WT plants. Unexpectedly, the levels of Met in the two sets of the transgenic seeds did not increase beyond the levels of WT seeds and were even significantly reduced (Figure 3; Additional file 2: Table S1). These results are unlike those reported for tobacco plants overexpressing these two forms of AtCGS, which show significantly higher levels of Met in their leaves $[11,12]$. In addition, these results also differ from those obtained from soybean and azuki bean seeds expressing the feedback-insensitive forms of AtCGS, which exhibit significantly higher levels of soluble Met (two- to six-fold higher compared to their corresponding WT seeds) $[9,10]$.

The observation that Met does not accumulate in the seeds of the transgenic lines beyond WT levels can be the result of an elevation in catabolic enzymes of Met. An increase in the expression of catabolic enzymes during seed development was reported in tobacco seeds expressing a key enzyme of lysine synthesis [24]. These seeds exhibit significantly higher lysine content compared to WT during their development; however, this triggers the expression of a lysine catabolic enzyme at a later stage of seed development, and eventually the level of lysine does not increase in the dry seeds [24]. We expected that if this phenomenon occurs also in the transgenic seeds expressing the two forms of AtCGS, the level of Met will be increased during seed development

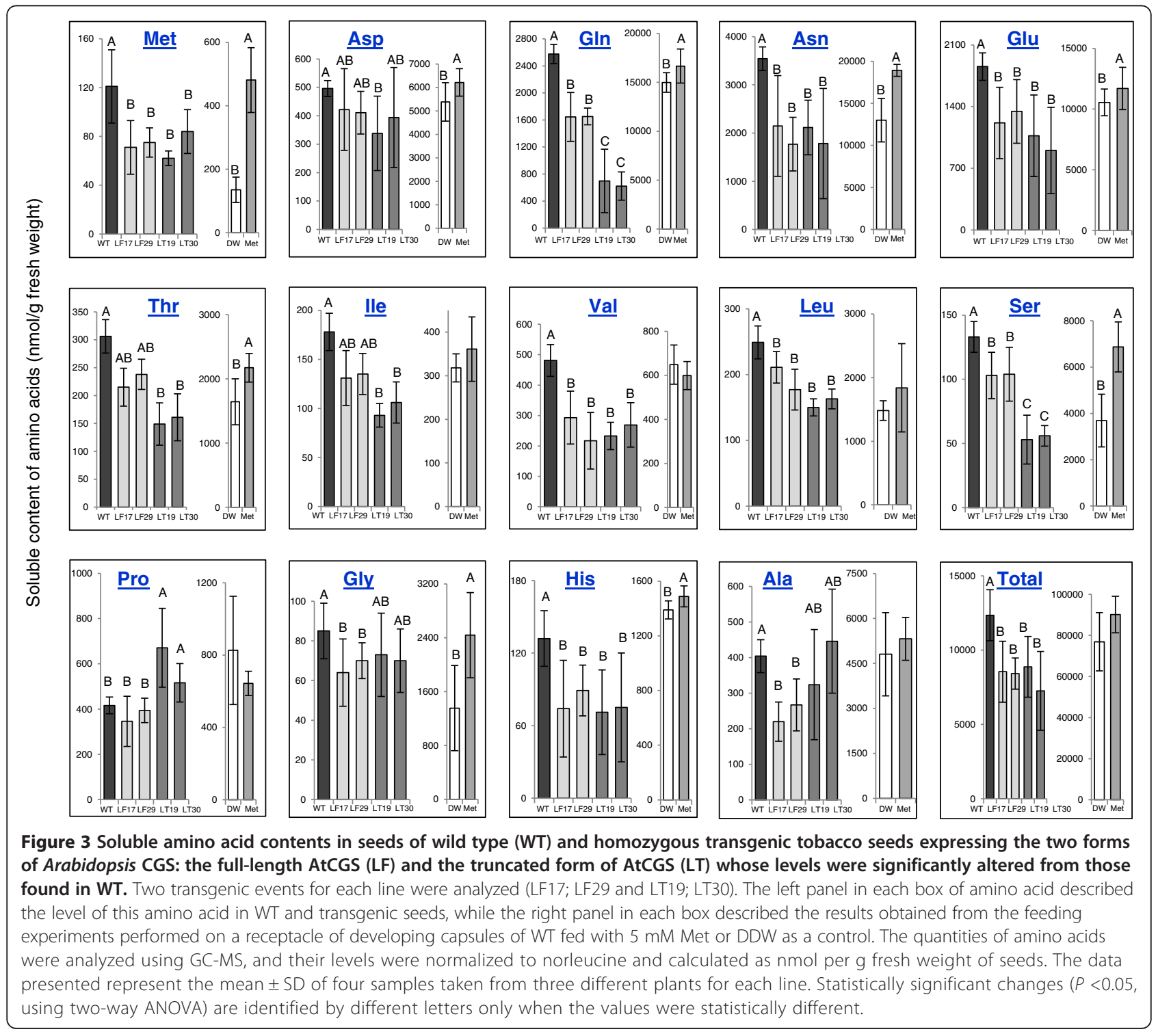


compared to WT seeds. To test this, the level of Met was measured during eight stages of seed development. The results show that the levels of soluble Met in the transgenic seeds are similar to those found in WT seeds during all developmental stages (shown for LT seeds, Additional file 1: Figure S3). This suggests that similar processes occur in the WT and the transgenic seeds during seed development, and that the low Met level found in dry transgenic seeds is apparently not related to an elevation in the rate of Met catabolic enzymes. However, in the future, when the sequences of the genes encoded to the two main catabolic enzymes of Met, SAM synthase and Met $\gamma$-lyase (Figure 1) from tobacco will be discovered, it will be worthwhile studying if their expression levels and/or activities increase during seed development in the transgenic lines. In addition, the role of the Young cycle and the methyl cycle derived from Met could be studied in these seeds to gain better knowledge of these pathways when the expression level of AtCGS increased in seeds.

\section{Evidence of enhanced Met synthesis in transgenic seeds during seed development}

The synthesis of Met is connected with other amino acids such as those belonging to the aspartate family pathway (Figure 1). Since the reduction of soluble Met in the transgenic seeds was unexpected, we have measured the levels of other amino acids to define changes that occur with an increase in expression level of AtCGS. The analysis revealed that in addition to Met, the levels of seven amino acids: glutamine, glutamate, asparagine, serine, valine, leucine and histidine were significantly reduced in both transgenic lines. The levels of threonine, and isoleucine were reduced significantly only in transgenic LT seeds, while alanine and glycine were reduced significantly only in LF seeds. The level of proline increased significantly in LT seeds, compared to WT seeds (Figure 3; Additional file 2: Table S1). From these altered amino acids, eight can be considered as Met-related amino acids (Figure 1). Glutamine and glutamate serve as precursors for aspartate that donates its carbon-amino acid skeleton to Met synthesis. The levels of these amino acids tend to be reduced when the content of Met, as well as levels of lysine and threonine, two additional amino acids that belong to the aspartate family of amino acids (Figure 1), increase [12,24,25]. Threonine competes with Met for their common precursor $O$-phosphohomoserine (Figure 1), and a decrease in the levels of threonine was observed in several plants following an increase in Met levels [1]. The branched amino acids, leucine, isoleucine and valine, are related to Met since the carbon/amino skeleton of these amino acids, is generated by the catabolism of threonine and Met [26-28]. Indeed, their levels increased significantly when high levels of Met were found in the leaves of transgenic tobacco and Arabidopsis plants [12,29]. Serine is the precursor of the carbon/ amino skeleton for cysteine synthesis, which is the sulfur donor for Met synthesis. A decrease in serine content was reported when the requirement for cysteine and Met increased [30-32]. The decrease in the level of serine suggests that the level of cysteine was decreased as well. Thus, an HPLC analysis was performed to measure the level of cysteine. The results show that the level of cysteine decreased significantly $(\mathrm{p}<0.05)$ in LT seeds, while in LF seeds, the

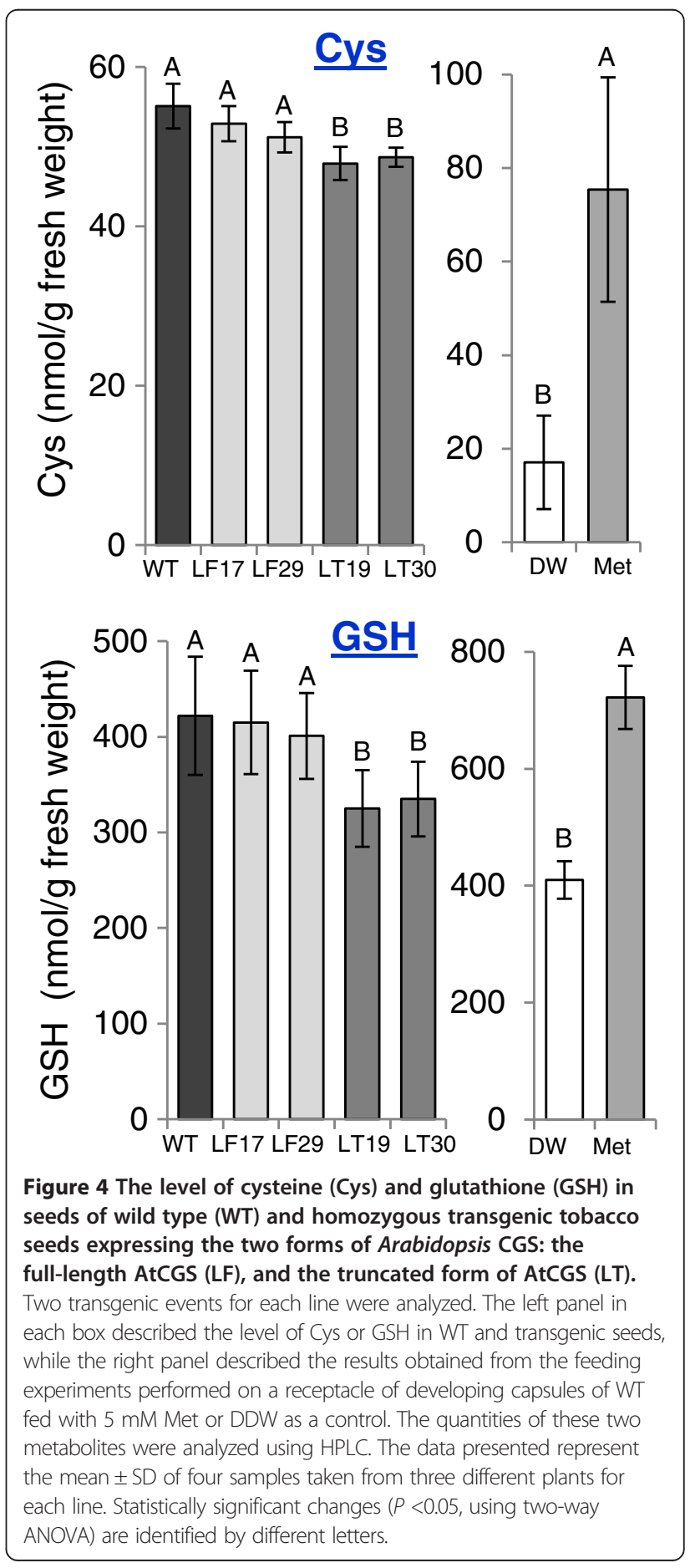


decrease in cysteine levels was insignificant (Figure 4). Since cysteine is also used as a precursor for the synthesis of glutathione (GSH), and the level of GSH is limited by cysteine (e.g., [33-35]), the level of GSH was also determined. Similarly to cysteine, the level of GSH was insignificantly altered in LF, and reduced significantly in LT compared to WT (Figure 4), a reduction of $22 \%$.

The low levels of the 11 amino acids (including cysteine) in seeds of LT suggest that although we did not detect an increase in the amounts of soluble Met, the flux towards Met synthesis was enhanced in the transgenic seeds, causing a decrease in the content of Met precursors and competitors.

To test this assumption, and to assess whether the 11 amino acids whose levels were reduced in LT seeds, are indeed related to Met synthesis and metabolism, the receptacles of developing capsules of WT plants were fed with Met. It is expected that feeding with Met will reduce the flux towards Met synthesis due to a reduction in the expression level of NtCGS (Figure 2A). If this occurs, we will expect an elevation in the levels of Met precursors (such as aspartate, glutamine and cysteine) or Met competitors (threonine) in the seeds. To test this assumption, receptacles of developing capsules (16-17 days after flowering) were fed with $0.5 \mathrm{ml}$ of $5 \mathrm{mM}$ Met, or with DDW as a control. At this stage of development, the proteins accumulate and amino acids are synthesized in the seeds [16]. Twenty-four hours later, the seeds were collected from these capsules and the levels of free amino acids were analyzed. The values obtained for the soluble amino acids (nmol/g fresh weight) were significantly higher in seeds obtained from the fed capsules compared to those obtained from the dry seeds (comparing the value in each amino acid between the left and right panels in Figure 3). This apparently happens because at that stage of seed development, most of the amino acids are not yet incorporated into the seed-storage proteins.

The results show that the level of Met increased significantly in the seeds from capsules fed with Met (3.5-fold) (Figure 3; Additional file 2: Table S2), indicating that Met can be transported from the receptacle to the seeds. The level of aspartate, glutamate, glutamine, serine (Met precursors), threonine (Met competitor), glycine and histidine were increased significantly in the seeds fed with Met (Figure 3; Additional file 2: Table S2). The levels of homocysteine and homoserine, intermediate metabolites of the Met biosynthesis pathway (Figure 1), also increased significantly (Additional file 2: Table S2), suggesting that under these conditions the activity of Met synthase, the last enzyme of Met synthesis, and homoserine kinase, are regulating the Met synthesis. In addition to these Metrelated amino acids, the level of lysine that belongs to the aspartate family together with Met also increased significantly, while the level of tryptophan decreased significantly
(Figure 3; Additional file 2: Table S2). The level of cysteine that was determined by HPLC shows that its level increased significantly in those fed with Met compared to those fed with DDW (a 4.4-fold increase). The level of GSH also increased significantly in these seeds at an elevation of 1.7-fold (Figure 4). These results, as well as those shown in Figure 4, suggest that a high expression of T-AtCGS increases the flux of cysteine towards Met synthesis in LT seeds at the expense of cysteine and its tripeptide, GSH.

The alternation in the content of cysteine and GSH in LT and in the Met-fed seeds was unexpected. Thus, to gain more knowledge about these changes, we studied how a high expression of T-AtCGS in LT seeds affects the transcript expression level of two genes in the GSH synthesis. We also determined the transcript level of adenosine- 5 ' -phosphosulfate-reductase, a key enzyme of the cysteine biosynthesis pathway [36,37]. None of the expression levels of these transcripts were altered between WT and LT seeds (Additional file 1: Figure S4). These results suggest that the alternation in GSH content is controlled less by the transcript levels of genes involved in cysteine and GSH biosynthesis pathways, and more by the flux of cysteine towards GSH and/or Met biosynthesis pathways. However, further studies are required to define the role of other factors and enzymes, as well as the role of $O$-acetylserine, the key metabolite that regulates the synthesis of cysteine [36].

Taken together, the data obtained by feeding experiments showed that the reduction of the flux towards Met synthesis caused by Met-feeding, led to an elevation in the levels of Met precursors and its competitors. These results support the assumption that although the level of Met in the transgenic seeds is relatively low, there is an enhanced flux towards Met synthesis during seed development. This is more pronounced in LT than in LF seeds that are feedback-sensitive to Met/SAM [15]. However, additional studies are required to monitor directly the metabolic flux leading to Met synthesis and the effect of T-AtCGS expression on this flux in seeds. The results also indicate that in tobacco seeds there is a connection between high expression levels of T-AtCGS to the levels of 12 amino acids and GSH.

\section{LT seeds contain higher levels of total Met in their proteins}

The results described above suggest that there is a higher flux towards Met synthesis in LT seeds. Since the results described in Additional file 1: Figure S3 imply that Met is converted to other metabolites in LT seeds at the same rate as it occurs in WT seeds, we assume that in LT seeds, Met was used mainly to synthesize storage proteins. Support of this assumption was derived from the observation that the soluble levels of the three branched amino acids that can be produced through Met catabolism [26,27] 
were not increased and even reduced significantly in LT seeds (Figure 3; Additional file 2: Table S1).

To test the assumption that in LT seeds Met was used to synthesize the seed's storage proteins, the levels of amino acids were measured after protein hydrolysis in dry seeds of WT, LF and LT. The total Met content in seeds of the two LF lines did not change significantly and were even slightly reduced when compared to WT (Table 1). One possible explanation for this observation is that the Met content in LF seeds cannot be increased further because of the feedback inhibition regulation occurring on AtCGS transcript level by SAM [15]. Moreover, since the level of soluble Met was reduced significantly in LF seeds compared to WT (Figure 3), it also implies that the sensitivity to the feedback inhibition of AtCGS (which is the major form of CGS in transgenic seeds) is higher than the NtCGS, hence reducing the ability of Met to accumulate. To test this assumption further, the expression level of NtCGS was measured in seedlings of WT and transgenic seedlings overexpressing the AtCGS [11]. As expected from the observation that the expression level of NtCGS is sensitive to a high level of Met (Figure 2A, left panel), its level was lower in seedlings overexpressing the F-AtCGS compared to WT (since they have a higher level of Met compared to WT in these plants) (Figure 2A). Next, we also analyzed the effect of a high content of Met on the expression level of AtCGS and NtCGS. The results (Figure 2A, B) showed that while the expression level of NtCGS was reduced by
$33 \%$, that of AtCGS was reduced by $52 \%$ compared to those fed with DDW. This suggests that AtCGS is more sensitive to feedback inhibition caused by a high level of Met compared to NtCGS. Since the sensitivity of AtCGS to Met is similar in the whole tissues of Arabidopsis [38], we expected that this is also the case for NtCGS. If this is indeed the case, the results described in Figure 2 can explain why the level of Met was reduced in LF transgenic seeds. However, further studies are required to test this assumption and to reveal why the contents of nine additional soluble amino acids (alanine, valine, leucine, glutamine, asparagine, glutamate, serine, glycine and histidine) were significantly decreased in LF transgenic seeds (Figure 3).

Unlike LF, LT seeds expressing the feedback-insensitive form of AtCGS (T-AtCGS) exhibit significantly higher levels of total Met, which increased 46 to $60 \%$, compared to WT seeds (Table 1). Based on the results obtained from LT, we suggest that the level of soluble Met in tobacco WT seeds limits the Met content that can be incorporated into proteins. Similar assumptions were made in several other studies showing that higher levels of soluble cysteine, lysine, tryptophan or Met in transgenic soybean seeds [10,39-41], and tryptophan in rice seeds [42] lead to an increase in these amino acids in the seeds' proteins. However, the ability of the higher soluble amino acids to incorporate into proteins appears to differ between different seeds. Significantly higher levels of soluble Met (about 6-fold) led to higher total Met (about

Table 1 The level of total amino acids in dry wild type (WT) seeds, in seeds expressing F-AtCGS (LF) and in seeds expressing T-AtCGS (LT), after protein hydrolysis

\begin{tabular}{lccccc}
\hline & WT & LF 29 & LF 17 & LT 19 & LT 30 \\
\hline Alanine & $19232 \pm 1957$ & $20778 \pm 2289$ & $21704 \pm 1098$ & $22891 \pm 1713$ & $21993 \pm 1665$ \\
Valine & $41269 \pm 6044$ & $41332 \pm 6002$ & $41269 \pm 3322$ & $43454 \pm 1418$ & $45885 \pm 3045$ \\
Serine & $18725 \pm 1181 \mathrm{~B}$ & $18090 \pm 3097 \mathrm{~B}$ & $18725 \pm 2660 \mathrm{~B}$ & $28859 \pm 130 \mathrm{~A}$ & $22908 \pm 127 \mathrm{~A}$ \\
Leucine & $45675 \pm 7705$ & $45499 \pm 5528$ & $45531 \pm 6186$ & $53449 \pm 985$ & $55274 \pm 1937$ \\
Threonine & $23410 \pm 4732$ & $23187 \pm 4212$ & $23410 \pm 3357$ & $34249 \pm 1026$ & $31440 \pm 2247$ \\
Isoleucine & $30985 \pm 4603$ & $31254 \pm 1233$ & $31981 \pm 1966$ & $32913 \pm 599$ & $39781 \pm 1098$ \\
Proline & $11234 \pm 1891 \mathrm{~B}$ & $10954 \pm 985 \mathrm{~B}$ & $11164 \pm 1550 \mathrm{~B}$ & $13196 \pm 1439 \mathrm{~A}$ & $13784 \pm 726 \mathrm{~A}$ \\
Glycine & $108546 \pm 4480$ & $108756 \pm 3567$ & $109546 \pm 5814$ & $109494 \pm 6499$ & $112348 \pm 4972$ \\
Methionine & $4374 \pm 315 \mathrm{~B}$ & $3962 \pm 997 \mathrm{~B}$ & $4199 \pm 313 \mathrm{~B}$ & $6992 \pm 459 \mathrm{~A}$ & $6376 \pm 573 \mathrm{~A}$ \\
Aspartate & $39625 \pm 11058 \mathrm{~B}$ & $41500 \pm 9876 \mathrm{~B}$ & $40625 \pm 7303 \mathrm{~B}$ & $61360 \pm 2182 \mathrm{~A}$ & $48541 \pm 3687 \mathrm{~A}$ \\
Phenylalanine & $21500 \pm 4772$ & $23058 \pm 5562$ & $22003 \pm 3568$ & $27960 \pm 1025$ & $28166 \pm 2235$ \\
Glutamate & $3058 \pm 785 \mathrm{~B}$ & $2984 \pm 689 \mathrm{~B}$ & $3644 \pm 330 \mathrm{~B}$ & $9217 \pm 242 \mathrm{~A}$ & $5896 \pm 564 \mathrm{~A}$ \\
Histidine & $2725 \pm 666$ & $2769 \pm 1072$ & $2863 \pm 605$ & $3415 \pm 358$ & $3722 \pm 231$ \\
Lysine & $20263 \pm 4330$ & $22645 \pm 5562$ & $22094 \pm 4570$ & $25838 \pm 1138$ & $24113 \pm 2645$ \\
Tyrosine & $19841 \pm 3273$ & $20476 \pm 5797$ & $21195 \pm 3165$ & $24230 \pm 1115$ & $27420 \pm 971$ \\
Total & $411462 \pm 21890 \mathrm{~B}$ & $417244 \pm 18664 \mathrm{~B}$ & $419953 \pm 11284 \mathrm{~B}$ & $497517 \pm 19064 \mathrm{~A}$ & $487647 \pm 13687 \mathrm{~A}$ \\
\hline
\end{tabular}

The levels of amino acids were measured using GC-MS and displayed as nmol/gr dry seeds. Values are representative of two independently grown sets of plants are the mean \pm standard deviation of five biological repetitions of $50 \mathrm{mg}$ seeds isolated from three plants per line. Statistically significant changes ( $<<0.05$, using two-way ANOVA) are identified by letters. 
2-fold) in soybean seeds of Zigongdongdou cultivar [10]. Yet, when the soybean cultivars Misuzudaizu and Bert were used, it was reported that although they exhibit significantly higher levels of soluble Met (2-fold), the level of total Met was not altered significantly [9]. Similarly, high levels of soluble threonine (16-fold) in tobacco seeds [24] and high levels of cysteine in lupine seeds (26-fold) [43] do not alter the total threonine or cysteine in the transgenic seeds $[43,44]$.

\section{LT seeds have higher levels of total amino acids and proteins}

In addition to the significantly higher levels of total Met in LT seeds, the total levels of aspartate, glutamate and two stress-associated amino acids, proline and serine also increased significantly in LT seeds (Table 1). Furthermore, the levels of most other amino acids in LT seeds were increased, although insignificantly. As a result, the total amino acids content increased significantly in LT seeds by $18-21 \%$ (Table 1). This elevation can explain why the levels of total soluble amino acids decrease significantly (Figure 3; Additional file 2: Table S1). A similar trend of higher total amino acids was also found in two transgenic soybean seeds expressing the feedback-insensitive form of AtCGS, in which the level of total amino acids increased by $55 \%$ and $76 \%$ [10]. However, this is not a general phenomenon in other amino acids, since transgenic soybean seeds having higher levels of total tryptophan and Arabidopsis seeds having a higher level of lysine did not show an increase in total amino acids levels [40,45]. This suggests that Met level is a limiting factor of protein synthesis in tobacco seeds, and that when Met synthesis is enhanced, most of the other soluble amino acids are able to incorporate into proteins. However, further studies are required to define the mechanism behind this phenotype and to determine if an elevation in the content of other yet unstudied amino acids can also lead to the same phenomenon.

The increase in the amount of protein-incorporated amino acids suggests that LT seeds have higher protein content. To test this possibility, the amounts of water-soluble proteins in the four samples of seeds were measured from groups of $10 \mathrm{mg}$ seeds from each line. The results revealed that $\mathrm{LT}$ seeds have a higher content of proteins $(2.22 \pm 0.36 \mu \mathrm{g} / \mu \mathrm{l}$ in $\mathrm{LT}$ compared to $1.74 \pm 0.39 \mu \mathrm{g} / \mu \mathrm{l}$ in WT, an increase of $27 \%$, which is statistically insignificantly).

\section{The effect of higher expression levels of T-AtCGS on the seeds' primary metabolites}

The expression of T-AtCGS leads to an elevated level of total Met (Table 1) and altered levels of 12 soluble amino acids (Additional file 2: Table S1). In order to assess whether the higher flux towards Met synthesis is associated with additional changes in the levels of primary metabolites, we performed a metabolic profiling analysis using established gas chromatography-mass spectrometry (GC-MS) [46-48]. Despite the significant changes in seed metabolism that occur during seed development, little is known regarding changes to the metabolic profile of the seed during development when heterologous genes are expressed $[17,18]$. Therefore, we were interested in studying whether a perturbation in Met metabolism influences individual metabolites within the seeds.

The GC-MS analyses revealed 102 peaks having a significantly higher signal-to-noise value. In order to identify the chemical nature of as many peaks as possible, standards were used. In addition, the spectra of all the peaks were compared with commercially available electron mass spectrum libraries, NIST and WILEY. Fiftytwo annotated specific compounds were detected in WT and transgenic seeds (Additional file 3: Table S4), several of which appeared at more than one retention time.

When analyzing the trends of the changes to metabolites concentration in seeds by principal component analysis (PCA), the WT and LT genotypes exhibited clearly distinguishable differences, implying a relatively strong effect of the genetic manipulation on the primary metabolism of LT seeds (Figure 5). These results are dissimilar to those reported in Arabidopsis seeds having higher levels of lysine, which show little differences from WT [17]. The PCA analysis allowed us to define the metabolites and peaks that contributed significantly to the variance between WT and LT genotypes. Thus, these metabolites and peaks were also assessed by a two-way ANOVA.

Among the 52 annotated metabolites, the levels of seven metabolites were significantly higher in LT compared to WT seeds: glycerol, gluconic acid, galacturonic acid, xylitol, lyxonic acid, melibiose and adenosine, while that of pantothenic acid was reduced significantly (Figure 6). In addition, four peaks (17, 31, 72, 73; Additional file 3: Table S4), decreased significantly in LT seeds, while two others $(90,93)$ increased significantly. None of the annotated metabolites are known according to the literature to be related to Met. Most of the compounds whose levels increased in LT are sugars or their oxidized derivatives. Gluconic acid is the oxidized form of glucose, and galacturonic acid, which is the main component of pectin, is an oxidized form of galactose. Xylitol and lyxonic acid (also called xylonite) are produced from xylose by hydrogenation or mild oxidation, respectively. The higher levels of the oxidized metabolites, as well as higher levels of soluble proline (Figure 3; Additional file 2: Table S1), and total level of serine, which are stress induced amino acids $[49,50]$, imply that oxidative stress occurs during seed development of LT seeds.

In addition to these sugars and their derivatives, the level of adenosine (a purine nucleoside) also increased. Adenosine is known to elicit numerous physiological 


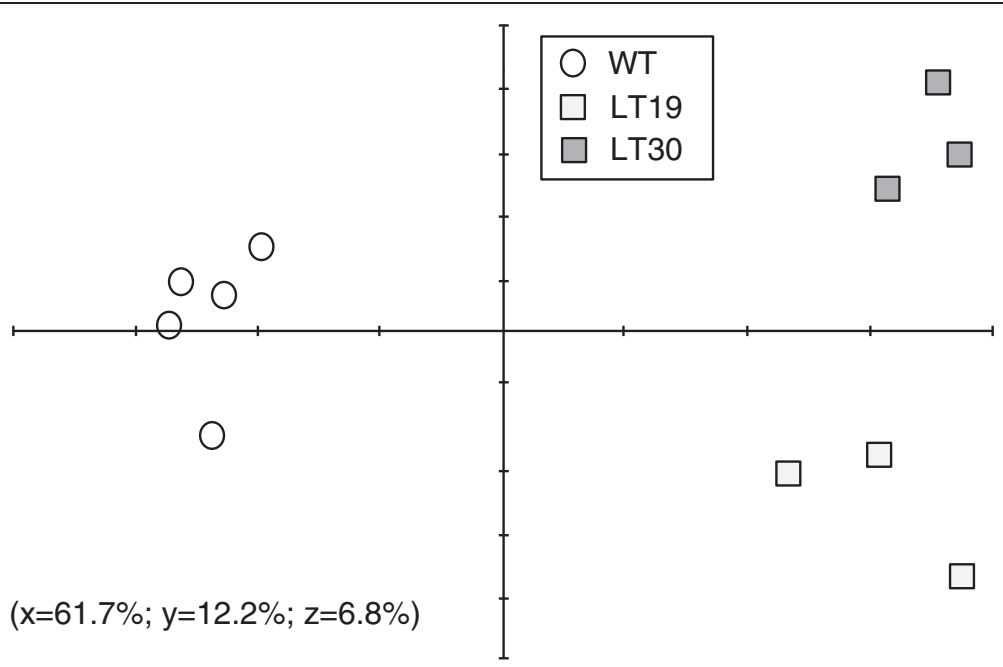

Figure 5 Principal component analysis (PCA) of 102 peaks that represent different metabolites measured in wild-type (WT) and LT transgenic seeds expressing the T-AtCGS gene. PCA is presented as a combination of the first three dimensions, which together comprise $80.7 \%$ of the metabolite variance. Each data point represents an independent sample. The data analysis was performed using TMEV software. Component 1 explained $61.7 \%$ of the variance, component $212.2 \%$, and component $36.8 \%$. The combined percentages of the variance are given in brackets.

responses in plants [51,52], and is required for the synthesis of the main Met metabolite, SAM. Purines require folic acid for their synthesis, which is also necessary for the synthesis of methyl-tetrahydrofolate, the substrate for Met synthase, the last enzyme of Met synthesis. However, this is a putative link and further studies are required to reveal this link between Met to adenosine.

While the levels of these metabolites were increased, the content of pantothenic acid (vitamin $\mathrm{B}_{5}$ ) was reduced in LT seeds. Pantothenic acid is the precursor of coenzyme A and is synthesized from aspartate [53], the carbon/amino donor of Met. Thus, a decrease in soluble aspartate levels in LT seeds (Additional file 2: Table S1) might leave less aspartate available for the synthesis of pantothenic acid. Another putative link between Met and pantothenic acid is that they both require folatedependent enzymes for their synthesis [54]. Thus, when the level of Met increases (and also adenosine), less folate

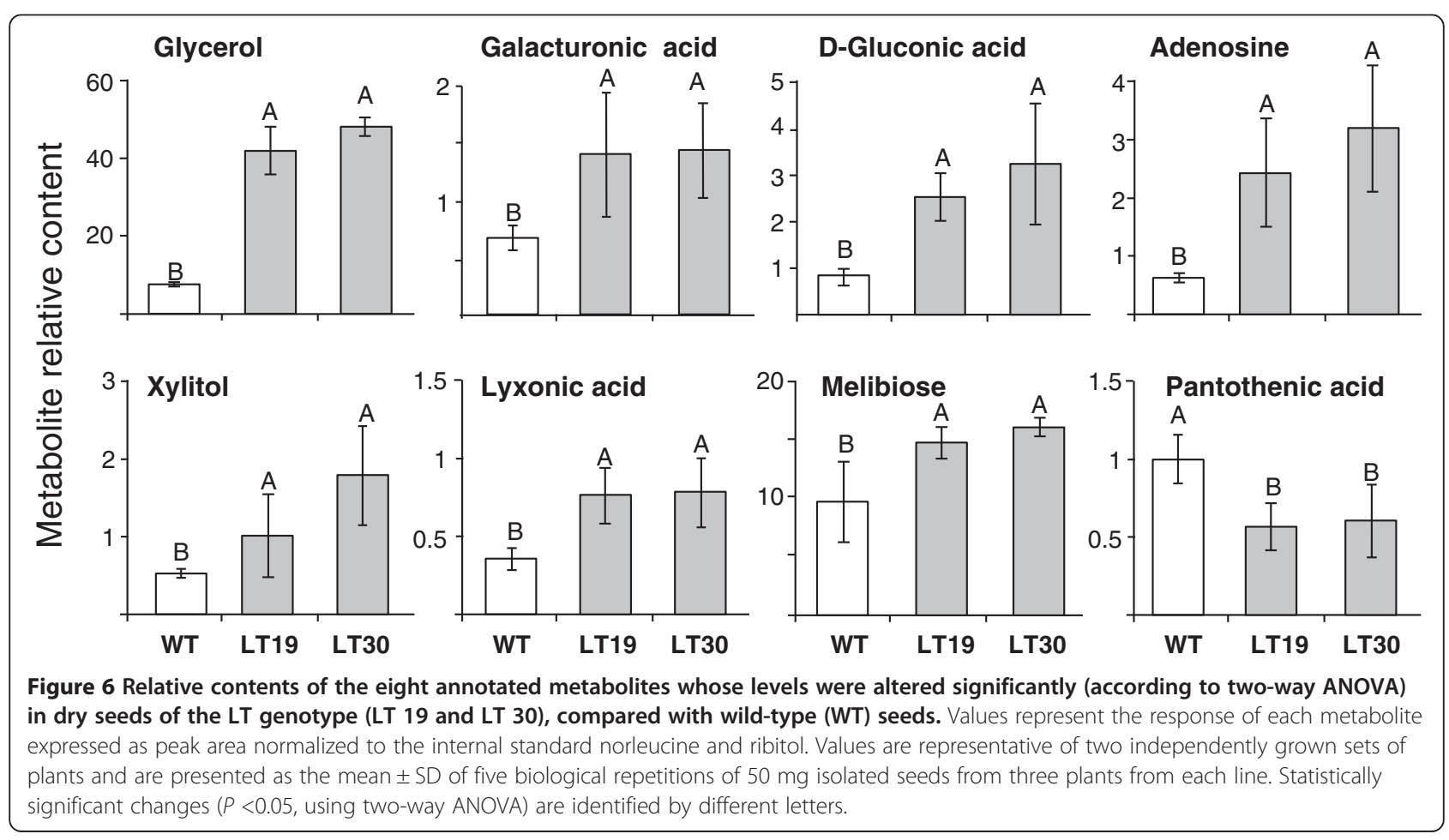


is available for the synthesis of pantothenic acid. However, again further studies are required to clarify these relationships.

To better understand the relationships between these metabolites and the Met metabolism, the WT seeds whose developing capsules were fed with Met were analyzed using the GC-MS scan method. This allowed us to detect the metabolites whose levels were altered $24 \mathrm{~h}$ after feeding. The results show that the levels of xylitol and melibiose increased significantly in seeds fed with Met (32\% and 27\%, respectively). The level of adenosine increased by $15 \%$ but was statistically insignificant. The levels of the other compounds did not change significantly compared to seeds fed with DDW. Most probably, the short period of exposure to high Met level is not enough to alter the contents of these metabolites in a significant way. However, this analysis strengthens the assumption that a link exists between xylitol and melibiose to high expression levels of AtCGS in tobacco seeds and to higher level of Met.

\section{The level of lipids and total reducing sugars in LT seeds}

The significant increase in glycerol content in LT seeds may affect lipids content, since glycerol is a precursor for triacylglycerol and may play a role in regulating oil content in seeds $[55,56]$. In addition, the higher level of protein in these seeds may also affect the level of lipids and starch, as was previously reported for soybean seeds [57]. We have used the Soxhlet method to measure oil content. This analysis revealed that the oil content of LT seeds is slightly higher than that of WT, but this increase was statistically insignificant $(40.71 \% \pm 2.46$ and $36.75 \% \pm 2.29$, respectively).

Since the level of total amino acids in proteins had increased in LT seeds and the level of oil had decreased slightly, we also measured the level of total reducing sugars following carbohydrate hydrolysis, which represents starch content. The content of reducing sugars was slightly but significantly lower in LT seeds than in WT seeds $(10.65 \pm 0.31$ and $11.84 \pm 0.13 \mathrm{mg}$ per $1 \mathrm{~g}$ dry seeds, respectively). This suggests that the higher protein content was at the expense of starch accumulation. This decrease in total reducing sugars may also be associated with the observation that besides amino acids, most of the metabolites whose levels were altered significantly in LT seeds were sugars and their derivatives (Figure 6). A putative link is that the carbon skeleton of glucoses was modified to other sugars, leaving less glucose available to be incorporated into starch.

\section{The germination rate of LT seeds is slightly reduced}

The extent of the changes in the contents of cellular constituents and key metabolites, which include 20 annotated primary metabolites (including amino acids), total proteins, lipids and reducing sugars in LT seeds, may affect the seeds' phenotype and their germination rate. Thus, we compared the morphological phenotypes of WT and LT seeds and found no significant differences. The average weight of $100 \mathrm{LT}$ seeds decreased compared to WT seeds $(87.5 \pm 0.64 \mathrm{mg}$ in LT compared to $93.6 \pm$ $1.19 \mathrm{mg}$ in WT seeds), but this decrease was statistically insignificant. LT seeds had a lower germination rate compared to WT five days after imbibition (Additional file 1: Figure S5), however, four days later, the growth of WT and LT seedlings was indistinguishable. A decrease in germination rate of soybean and Arabidopsis seeds having increased levels of lysine was reported [41,45], however, that of soybean with an increased level of threonine [58] or Met [10] was not affected. Hence, different seeds respond in different ways to a high flux or to high level of soluble amino acids. In general, the results of the current study suggest that the germination rate of tobacco seeds is relatively tolerant to the perturbations that occur when the Met synthesis increases, causing significant changes to the profile of 20 annotated metabolites, as well as in the levels of total reducing sugars and proteins.

\section{Conclusions}

Based on the results described above, we propose the following explanation for the changes that occur in tobacco seeds following the expression of AtCGS: a high expression level of the Met/SAM feedback-insensitive form of AtCGS in LT seeds, unlike the feedback-sensitive form of AtCGS in LF seeds, leads to a significant increase in flux towards Met synthesis. This leads to a significant increase of Met synthesis, that causes a reduction to the contents of amino acids that serve as precursors for Met. The increase of Met synthesis enhanced the protein synthesis rate, which can utilize other soluble amino acids for incorporation into storage proteins during seed maturation. As a result, the amount of total amino acids increased (Table 1), as well as the amount of total water soluble proteins (by about 27\%). This suggests that CGS plays a role in Met synthesis in tobacco seeds as was found in legumes seeds $[9,10,59]$. However, further studies are required to assess whether Met can also be synthesized from SMM at the latter stages of seed development, as was suggested for Medicago truncatula [60].

In addition to our goal to assess the role of AtCGS in Met synthesis in seeds, we also aimed at deciphering how this manipulation cross-interacts with other primary metabolites in the seeds. The results show that the levels of 20 annotated primary metabolites (including amino acids) were altered in LT in comparison to WT seeds. These include 12 metabolites whose levels decreased and 8 metabolites whose levels increased. The results also indicate that unlike lysine metabolism, Met metabolism has a relatively large impact on primary metabolites, since high lysine content affected only four metabolites connected to the 
TCA cycle [17]. In general, the levels of most free amino acids were reduced in LT, while the levels of sugars and their derivates were elevated. The increase in oxidized sugars and the higher levels of total proline and serine, two amino acids that their level increase during oxidative stress $[49,50]$, suggest that LT seeds suffer from oxidative stress. The oxidative stress might be related to the low content of GSH, a major antioxidant compound [61], in LT seeds. However, further studies are required to clarify the relations between high level of T-AtCGS, low GSH and oxidative stress, during seed development.

This study provides information on the metabolic consequences of enhancing the expression level of CGS is in seeds. In addition, since the levels of total soluble proteins increase in LT seeds by $27 \%$, the total lipid content was reduced by about $4 \%$ (although insignificantly), and the total levels of reducing sugars, which represent starch content, was reduced significantly by $4.6 \%$, we also define that the high expression level of T-AtCGS affects the seed's nutritional quality.

\section{Methods \\ Generation of transgenic plants expressing different forms of AtCGS in a seed-specific manner}

The binary constructs were prepared as described in Song et al. [10] and in Additional file 1: Figure S1. Tobacco plants (Nicotiana tabacum cv Samsun NN) were transformed with these constructs as previously described [11]. The plants were grown in a growth chamber under 16/ 8 hours light/dark cycle and temperature of $23-27^{\circ} \mathrm{C}$. Heterozygous mature $\mathrm{T}_{0}$ seeds from 30 kanamycin-resistant plants from each transgenic line were screened for protein expression level by immunoblot analysis [11]. Two plants having the highest expression levels from each set were chosen for further study. These plants were self-pollinated to obtain homozygous plants.

\section{Extraction, derivatization and analysis of seed amino acids}

Seeds from at least 15 capsules collected from each of the transgenic and WT lines were pooled. Free amino acids were extracted from $20 \mathrm{mg}$ of dry seeds. For total amino acid determination including protein-bound amino acids, $10 \mathrm{mg}$ of dry seeds were taken as described [30]. For acid hydrolysis, a Carousel 12 plus reaction station (Radleys, UK) was used. The amino acids were detected using the single ion method (SIM) of GC-MS, as previously described [30]. For cysteine and GSH determination, Dry seeds (50 mg) were ground with a mortar and pestle and then extracted and analyzed by HPLC, as previously described [33].

\section{GC-MS instrumentation and data analysis of primary metabolites using GC-MS}

For primary metabolites analysis, samples were prepared as described for the free amino acids [30] and $7 \mu \mathrm{l}$ of a retention time standard mixture $(0.2 \mathrm{mg} / \mathrm{ml}$ n-dodecane, n-pentadecane, n-nonadecane, n-docosane and n-octacosane, in pyridine); in addition, $4.6 \mu \mathrm{l}$ of a retention time standard mixture of norleucine and ribitol $(2 \mathrm{mg} / \mathrm{ml})$ were added prior to trimethylsilylation.

Samples were run on a GC-MS system (Agilent 7890A series GC system coupled with Agilent 5975c Mass Selective Detector), and a Gerstel $^{\oplus}$ multipurpose sampler (MPS2) was installed on this system. The analysis method was adapted to our system based on Roessner et al. (2001) [46]. Helium was used as the carrier gas at a flow rate of $1 \mathrm{~mL}$ per min. $1 \mu \mathrm{L}$ was injected in the splitless mode, the inlet temperature was $230^{\circ} \mathrm{C}$ and the pressure was 8.805 psi. The GC was performed on a $30 \mathrm{~m}$ RXI-5-Sil MS Capillary Column with a $0.25 \mathrm{~mm}$ inner diameter and $0.25 \mu \mathrm{m}$ film thickness (Restek, USA). The analysis was performed under the following temperature program: $5 \mathrm{~min}$ of isothermal heating at $70^{\circ} \mathrm{C}$ followed by a $5^{\circ} \mathrm{C} \min ^{-1}$ oven temperature gradient to $310^{\circ} \mathrm{C}$, and a final $3 \mathrm{~min}$ of heating at $310^{\circ} \mathrm{C}$. The system was then temperature equilibrated for $6 \mathrm{~min}$ at $70^{\circ} \mathrm{C}$ before the injection of the next sample. Mass spectra were recorded at $2 \mathrm{scan} \mathrm{sec}^{-1}$ with a scanning range of 50 to $600 \mathrm{~m} / \mathrm{z}$.

Standards substances (most of them kindly provided by Asaph Aharoni, Weizmann Institute) were dissolved in $50 \%$ methanol $(1 \mathrm{mg} / \mathrm{ml})$. A $5 \mu \mathrm{l}$ volume of standard solution was dried under vacuum and derivatized with $40 \mu \mathrm{l}$ of $20 \mathrm{mg} / \mathrm{ml}$ methoxyamine hydrochloride in pyridine and $100 \mu \mathrm{l}$ N-methyl-N-(trimethylsilyl) trifluoroacetamide (MSTFA). The data collected were obtained using Agilent GC/MSD Productivity ChemStation software. All peaks above the baseline threshold were quantified and grouped according to retention time, with areas normalized to norleucine and ribitol. Substances were identified by comparison with standards, and were also compared with commercially available electron mass spectrum libraries NIST and WILEY.

\section{Application of Met to developing capsules}

Half $\mathrm{ml}$ of $5 \mathrm{mM}$ Met in sterile DDW was injected into the receptacle of developing capsules of WT 16-17 days after flowering. At this stage, seed color turned from white to light brown. After $24 \mathrm{hr}$, the capsules were harvested and kept on ice. Seeds were separated from the capsules, dried using a lyophilizer and stored at $-70^{\circ} \mathrm{C}$ until used. Capsules injected with DDW were used as control.

\section{RNA extraction from tobacco seedlings and expression analysis}

For RNA extraction, plants were grown in Nitsch agar plates [Nitsch medium (DUCHEFA), $\mathrm{pH}$ adjusted to 5.8 with $\mathrm{KOH} 0.9 \%(\mathrm{w} / \mathrm{v})$ containing $2 \%$ sucrose] at light/ dark cycle (16 h/8 h). Twenty-one-day-old tobacco seedlings were fed with $5 \mathrm{mM}$ Met or with DDW as a 
control. After $6 \mathrm{~h}$, plants were frozen in liquid nitrogen as a pool of 30 seedlings in five biological repeats and kept until use at $-80^{\circ} \mathrm{C}$. For qRT-PCR, the RNA was extracted and cDNA was synthesized as previously described [10]. Primes used in the qRT-PCR analysis are summarized in Additional file 2: Table S3. To normalize the variance among samples, the PP2A transcript level was used as endogenous control [62]. The values presented are the mean of three biological replicates.

\section{Determination of germination rates of transgenic LT and WT seeds}

Seeds were placed overnight in water at $12^{\circ} \mathrm{C}$ with gentle shaking and then transferred to Petri dishes with Watmann paper wetted with water. The plates were placed at $25^{\circ} \mathrm{C}$ in a standard plant growth chamber under a 16/8-h light/dark regime for 10 days.

\section{Total protein, lipid and reduced sugars determination in seeds}

For total protein determination, $10 \mathrm{mg}$ seeds were ground in $120 \mu \mathrm{l}$ of $25 \mathrm{mM}$ Na phosphate buffer $\mathrm{pH}=7.8$ with a protease inhibitor cocktail (Sigma, P9599). After two centrifugation cycles $\left(14,000 \mathrm{rpm} 4^{\circ} \mathrm{C}\right.$ for $\left.20 \mathrm{~min}\right)$, total protein was determined using a Bradford reagent (Bio-Rad Hercules, Calif). Bovine serum albumin was used as a standard.

The Soxhlet method was used for lipid determination as previously described [10]. Reducing sugars were measured after carbohydrates and starch hydrolysis calorimetrically using the Sumner method [63]. $150 \mathrm{mg}$ of seeds were dried and ground as described above. The seeds were extracted in $80 \mathrm{ml}$ of $\mathrm{HCl} 1 \mathrm{~N}$ at $70^{\circ} \mathrm{C}$ for $2 \mathrm{~h}$. After cooling, the $\mathrm{pH}$ was corrected to 7.5 and the volume corrected to $100 \mathrm{ml}$ with water. 0.1 and $0.2 \mathrm{ml}$ of the samples were then mixed with 1.9 and $1.8 \mathrm{ml}$ water, respectively, and $2 \mathrm{ml}$ of Sumner reagent was added. The reducing sugars were detected at $550 \mathrm{~nm}$.

\section{Statistical and network analyses}

The data obtained from this study were analyzed statistically using JMP 8 software. In this software, we used ANOVA and the Student's t-test programs, as described in the text. A P value of $<0.05$ was considered statistically significant.

For the statistical analysis required for the metabolic profile analysis, PCA and two-way ANOVA were performed on the datasets obtained from metabolite profiling with the software package TMEV [64] using the default weighted covariance-estimation function. Prior to the analysis, data were $\log$ transformed and normalized to the median of the entire sample set for each metabolite. To test statistical significance between specific time points, t-tests were performed using the algorithm incorporated into Microsoft
Excel. We set the correlation significance threshold fixed at the level of the Spearman correlation coefficient.

\section{Additional files}

Additional file 1: Figure S1. (a) Schematic presentation of Arabidopsis cystathionine $\gamma$-synthase (AtCGS) protein, and the constructs used in this study. Figure S2: Samples of $T_{1}$ screening of transgenic seeds LF and LT seeds by immunoblot analysis. Figure S3: The level of soluble Met in WT and transgenic LT seeds during seed development. Figure S4: Quantitative real-time PCR analyses of representative genes in the cysteine and GSH biosynthesis in LT and WT seeds. Figure S5: The germination rate of LT seeds.

Additional file 2: Table S1. Soluble amino acid contents in seeds of WT and homozygous transgenic tobacco seeds of LF and LT. Table S2: Soluble amino acid contents in WT tobacco seeds after application of double distilled water (DDW) or $5 \mathrm{mM}$ methionine in DDW, to the receptacle of developing capsules. Table S3: List of primers used in this study.

Additional file 3: Table S4. The entire data set of the relative contents of metabolites in WT, LF and LT seeds.

\section{Abbreviations}

Met: Methionine; SAM: S-adenosyl methionine; SMM: S-methyl methionine; CGS: Cystathionine $\mathrm{Y}$-synthase; AtCGS: Arabidopsis CGS; WT: Wild type; GSH: Glutathione; qRT-PCR: Quantitative real time PCR; GC-MS: Gas chromatography-mass spectrometry; DDW: Double distilled water.

\section{Competing interests}

The authors declare that they have no competing interests.

\section{Authors' contributions}

IM designed and performed the construction of the binary construct, the expression level analysis of the transgenic seeds, together with IG they analysed the amino acids content and performed the feeding experiments; $\mathrm{YH}$ carried out the molecular genetic studies in tobacco plants, and performed the statistical analysis; RA designed and coordinated the study and wrote the manuscript. All authors read and approved the final manuscript.

\section{Authors' information}

RA is Associated Professor at Tel Hai Collage and Migal Research Institute, Israel. In the last years she mainly studied the metabolism of Met in plants. Dr. YH, and IM are part of her research team. IG is a MSc. student.

\section{Acknowledgments}

We would like to thank Ulrich Wobus, Gatersleben Germany, who kindly provided the Legumin B4 promoter, Asaph Aharoni for the library of compounds for GC-MS analysis, Gidi Baum for his critical reading of the manuscript. We are deeply grateful to Prof. Rachel Galon from the Israel Academy of Science who promoted and encouraged this research. This work was supported by the Israel Science Foundation (ISF grant 231-09), and partly by research grant no. IS-4248-09 R from BARD, Yad Hanadiv Foundation (through the Israel Academy of Science) and UJIA, Great Britain Foundation.

Received: 29 September 2013 Accepted: 3 December 2013 Published: 7 December 2013

\section{References}

1. Amir R: Current understanding of the factors regulating methionine content in vegetative tissues of higher plants. Amino Acids 2010, 39(4):917-931.

2. Hesse H, Kreft O, Maimann S, Zeh M, Hoefgen R: Current understanding of the regulation of methionine biosynthesis in plants. J Exp Bot 2004, 55(404):1799-1808.

3. Ravanel S, Gakiere B, Job D, Douce R: The specific features of methionine biosynthesis and metabolism in plants. Proc Natl Acad Sci USA 1998, 95(13):7805-7812. 
4. Amir R, Han T, Ma F: Bioengineering approaches to improve the nutritional values of seeds by increasing their methionine content Mol Breed 2012, 29:915-924.

5. Galili G, Amir R: Fortifying plants with the essential amino acids lysine and methionine to improve nutritional quality. Plant Biotechnol J 2013 11(2):211-222

6. Hesse $H$, Hoefgen R: Molecular aspects of methionine biosynthesis. Trends Plant Sci 2003, 8(6):259-262.

7. Bourgis F, Roje S, Nuccio ML, Fisher DB, Tarczynski MC, Li C, Herschbach C, Rennenberg H, Pimenta MJ, Shen TL, et al: S-methylmethionine plays a major role in phloem sulfur transport and is synthesized by a novel type of methyltransferase. Plant Cell 1999, 11(8):1485-1498.

8. Lee M, Huang T, Toro-Ramos T, Fraga M, Last RL, Jander G: Reduced activity of Arabidopsis thaliana HMT2, a methionine biosynthetic enzyme, increases seed methionine content. Plant J 2008, 54(2):310-320.

9. Hanafy MS, Rahman SM, Nakamoto Y, Fujiwara T, Naito S, Wakasa K, Ishimoto M: Differential response of methionine metabolism in two grain legumes, soybean and azuki bean, expressing a mutated form of Arabidopsis cystathionine gamma-synthase. J Plant Physiol 2013, 170(3):338-345.

10. Song S, Hou W, Godo I, Wu C, Yu Y, Matityahu I, Hacham Y, Sun S, Han T, Amir R: Soybean seeds expressing feedback-insensitive cystathionine Y-synthase exhibit a higher content of methionine. J Exp Bot 2013, 64(7):1917-1926.

11. Hacham $Y$, Avraham T, Amir R: The N-terminal region of Arabidopsis cystathionine gamma-synthase plays an important regulatory role in methionine metabolism. Plant Physiol 2002, 128(2):454-462.

12. Hacham Y, Matityahu I, Schuster G, Amir R: Overexpression of mutated forms of aspartate kinase and cystathionine gamma-synthase in tobacco leaves resulted in the high accumulation of methionine and threonine. Plant J 2008, 54(2):260-271.

13. Hacham $Y$, Schuster $G$, Amir R: An in vivo internal deletion in the N-terminus region of Arabidopsis cystathionine gamma-synthase results in CGS expression that is insensitive to methionine. Plant J 2006, 45(6):955-967.

14. Hacham $Y$, Song L, Schuster G, Amir R: Lysine enhances methionine content by modulating the expression of S-adenosylmethionine synthase. Plant J 2007, 51(5):850-861.

15. Chiba $Y$, Sakurai R, Yoshino M, Ominato K, Ishikawa M, Onouchi H, Naito S: $\mathrm{S}$-adenosyl-L-methionine is an effector in the posttranscriptional autoregulation of the cystathionine gamma-synthase gene in Arabidopsis. Proc Natl Acad Sci USA 2003, 100(18):10225-10230.

16. Karchi H, Miron D, Ben-Yaacov S, Galili G: The lysine-dependent stimulation of lysine catabolism in tobacco seed requires calcium and protein phosphorylation. Plant Cell 1995, 7(11):1963-1970.

17. Angelovici R, Fait A, Zhu X, Szymanski J, Feldmesser E, Fernie AR, Galili G: Deciphering transcriptional and metabolic networks associated with lysine metabolism during Arabidopsis seed development. Plant Physiol 2009, 151(4):2058-2072.

18. Fait A, Nesi AN, Angelovici R, Lehmann M, Pham PA, Song L, Haslam RP, Napier JA, Galili G, Fernie AR: Targeted enhancement of glutamate-to-gamma-aminobutyrate conversion in Arabidopsis seeds affects carbon-nitrogen balance and storage reserves in a development-dependent manner. Plant Physiol 2011, 157(3):1026-1042.

19. Gallardo K, Thompson R, Burstin J: Reserve accumulation in legume seeds. C R Biol 2008, 331(10):755-762.

20. Chiba Y, Ishikawa M, Kijima F, Tyson RH, Kim J, Yamamoto A, Nambara E, Leustek T, Wallsgrove RM, Naito S: Evidence for autoregulation of cystathionine gamma-synthase mRNA stability in Arabidopsis. Science 1999, 286(5443):1371-1374.

21. Katz YS, Galili G, Amir R: Regulatory role of cystathionine-gamma-synthase and de novo synthesis of methionine in ethylene production during tomato fruit ripening. Plant Mol Biol 2006, 61(1-2):255-268.

22. Kreft $\mathrm{O}$, Hoefgen $\mathrm{R}$, Hesse $\mathrm{H}$ : Functional analysis of cystathionine gamma-synthase in genetically engineered potato plants. Plant Physiol 2003, 131(4):1843-1854

23. Baumlein $H$, Nagy I, Villarroel $R$, Inze D, Wobus U: Cis-analysis of a seed protein gene promoter: the conservative RY repeat CATGCATG within the legumin box is essential for tissue-specific expression of a legumin gene. Plant J 1992, 2(2):233-239.

24. Karchi H, Shaul O, Galili G: Lysine synthesis and catabolism are coordinately regulated during tobacco seed development. Proc Natl Acad Sci USA 1994, 91(7):2577-2581.
25. Zeh M, Casazza AP, Kreft O, Roessner U, Bieberich K, Willmitzer L, Hoefgen R, Hesse $\mathrm{H}$ : Antisense inhibition of threonine synthase leads to high methionine content in transgenic potato plants. Plant Physio/ 2001, 127(3):792-802.

26. Joshi $V$, Jander G: Arabidopsis methionine gamma-lyase is regulated according to isoleucine biosynthesis needs but plays a subordinate role to threonine deaminase. Plant Physiol 2009, 151(1):367-378.

27. Joshi $V$, Joung JG, Fei $Z$, Jander G: Interdependence of threonine, methionine and isoleucine metabolism in plants: accumulation and transcriptional regulation under abiotic stress. Amino Acids 2010, 39(4):933-947.

28. Rebeille F, Jabrin S, Bligny R, Loizeau K, Gambonnet B, Van Wilder V, Douce R, Ravanel S: Methionine catabolism in Arabidopsis cells is initiated by a gamma-cleavage process and leads to S-methylcysteine and isoleucine syntheses. Proc Natl Acad Sci USA 2006, 103(42):15687-15692.

29. Kusano M, Fukushima A, Redestig H, Kobayashi M, Otsuki H, Onouchi H, Naito S, Hirai MY, Saito K: Comparative metabolomics charts the impact of genotype-dependent methionine accumulation in Arabidopsis thaliana. Amino Acids 2010, 39(4):1013-1021.

30. Golan A, Matitiyho I, Avraham T, Badani H, Galili S, Amir R: Soluble methionine enhances accumulation of a $15 \mathrm{kDa}$ zein, a methionine-rich storage protein, in transgenic alfalfa but not in transgenic tobacco plants. J Exp Bot 2005, 56(419):2443-2452.

31. Nikiforova V, Kempa S, Zeh M, Maimann S, Kreft O, Casazza AP, Riedel K, Tauberger E, Hoefgen R, Hesse H: Engineering of cysteine and methionine biosynthesis in potato. Amino Acids 2002, 22(3):259-278.

32. Stiller I, Dancs G, Hesse H, Hoefgen R, Banfalvi Z: Improving the nutritive value of tubers: elevation of cysteine and glutathione contents in the potato cultivar White Lady by marker-free transformation. J Biotechnol 2007, 128(2):335-343.

33. Matityahu I, Kachan L, Bar Ilan I, Amir R: Transgenic tobacco plants overexpressing the Met25 gene of Saccharomyces cerevisiae exhibit enhanced levels of cysteine and glutathione and increased tolerance to oxidative stress. Amino Acids 2006, 30(2):185-194.

34. Noji M, Saito M, Nakamura M, Aono M, Saji H, Saito K: Cysteine synthase overexpression in tobacco confers tolerance to sulfur-containing environmental pollutants. Plant Physio/ 2001, 126(3):973-980.

35. Wirtz M, Hell R: Functional analysis of the cysteine synthase protein complex from plants: structural, biochemical and regulatory properties. J Plant Physiol 2006, 163(3):273-286.

36. Wirtz M, Droux M: Synthesis of the sulfur amino acids: cysteine and methionine. Photosynth Res 2005, 86(3):345-362.

37. Davidian JC, Kopriva S: Regulation of sulfate uptake and assimilation-the same or not the same? Mol Plant 2010, 3(2):314-325

38. Kim J, Lee M, Chalam R, Martin MN, Leustek T, Boerjan W: Constitutive overexpression of cystathionine gamma-synthase in Arabidopsis leads to accumulation of soluble methionine and S-methylmethionine. Plant Physiol 2002, 128(1):95-107.

39. Kim WS, Chronis D, Juergens M, Schroeder AC, Hyun SW, Jez JM, Krishnan HB: Transgenic soybean plants overexpressing O-acetylserine sulfhydrylase accumulate enhanced levels of cysteine and Bowman-Birk protease inhibitor in seeds. Planta 2012, 235(1):13-23.

40. Kita Y, Nakamoto $Y$, Takahashi M, Kitamura K, Wakasa K, Ishimoto M: Manipulation of amino acid composition in soybean seeds by the combination of deregulated tryptophan biosynthesis and storage protein deficiency. Plant Cell Rep 2010, 29(1):87-95.

41. Falco SC, Guida T, Locke M, Mauvais J, Sanders C, Ward RT, Webber P: Transgenic canola and soybean seeds with increased lysine. Biotechnology (N Y) 1995, 13(6):577-582

42. Wakasa K, Hasegawa H, Nemoto H, Matsuda F, Miyazawa H, Tozawa Y, Morino K, Komatsu A, Yamada T, Terakawa T, et al: High-level tryptophan accumulation in seeds of transgenic rice and its limited effects on agronomic traits and seed metabolite profile. J Exp Bot 2006, 57(12):3069-3078.

43. Tabe L, Wirtz M, Molvig L, Droux M, Hell R: Overexpression of serine acetlytransferase produced large increases in O-acetylserine and free cysteine in developing seeds of a grain legume. J Exp Bot 2010, 61(3):721-733.

44. Karchi H, Shaul O, Galili G: Seed specific expression of a bacterial desensitized aspartate kinase increases the production of seed threonine and methionine in transgenic tobacco. Plant J 1993, 3:721-727.

45. Zhu X, Galili G: Increased lysine synthesis coupled with a knockout of its catabolism synergistically boosts lysine content and also transregulates the metabolism of other amino acids in Arabidopsis seeds. Plant Cell 2003, 15(4):845-853. 
46. Roessner $U$, Luedemann A, Brust $D$, Fiehn $O$, Linke $T$, Willmitzer $L$, Fernie A: Metabolic profiling allows comprehensive phenotyping of genetically or environmentally modified plant systems. Plant Cell 2001, 13(1):11-29.

47. Less $H$, Angelovici $R$, Tzin V, Galili G: Principal transcriptional regulation and genome-wide system interactions of the Asp-family and aromatic amino acid networks of amino acid metabolism in plants. Amino Acids 2010, 39(4):1023-1028.

48. Fait A, Angelovici R, Less H, Ohad I, Urbanczyk-Wochniak E, Fernie AR, Galili G: Arabidopsis seed development and germination is associated with temporally distinct metabolic switches. Plant Physiol 2006, 142(3):839-854

49. Hayat S, Hayat Q, Alyemeni MN, Wani AS, Pichtel J, Ahmad A: Role of proline under changing environments: a review. Plant Signal Behav 2012 7(11):1456-1466.

50. Nikiforova VJ, Kopka J, Tolstikov V, Fiehn O, Hopkins L, Hawkesford MJ,

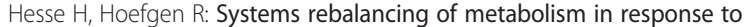
sulfur deprivation, as revealed by metabolome analysis of Arabidopsis plants. Plant Physiol 2005, 138(1):304-318.

51. Haferkamp I, Fernie AR, Neuhaus HE: Adenine nucleotide transport in plants: much more than a mitochondrial issue. Trends Plant Sci 2011, 16(9):507-515.

52. Ries S, Savithiry S, Wert V, Widders I: Rapid induction of lon pulses in tomato, cucumber, and maize plants following a foliar application of $\mathrm{L}$ (+)-adenosine. Plant Physiol 1993, 101(1):49-55.

53. Chakauya E, Coxon KM, Wei M, Macdonald MV, Barsby T, Abell C, Smith AG: Towards engineering increased pantothenate (vitamin $B(5)$ ) levels in plants. Plant Mol Biol 2008, 68(4-5):493-503

54. Mehrshahi P, Gonzalez-Jorge S, Akhtar TA, Ward JL, Santoyo-Castelazo A, Marcus SE, Lara-Nunez A, Ravanel S, Hawkins ND, Beale MH, et al: Functional analysis of folate polyglutamylation and its essential role in plant metabolism and development. Plant J 2010, 64(2):267-279.

55. Bates PD, Durrett TP, Ohlrogge JB, Pollard M: Analysis of acyl fluxes through multiple pathways of triacylglycerol synthesis in developing soybean embryos. Plant Physiol 2009, 150(1):55-72.

56. Vigeolas H, Waldeck P, Zank T, Geigenberger P: Increasing seed oil content in oil-seed rape (Brassica napus L.) by over-expression of a yeast glycerol-3-phosphate dehydrogenase under the control of a seed-specific promoter. Plant Biotechnol J 2007, 5(3):431-441.

57. Hernandez-Sebastia C, Marsolais F, Saravitz C, Israel D, Dewey RE, Huber SC: Free amino acid profiles suggest a possible role for asparagine in the control of storage-product accumulation in developing seeds of lowand high-protein soybean lines. J Exp Bot 2005, 56(417):1951-1963.

58. Qi Q, Huang J, Crowley J, Ruschke L, Goldman BS, Wen L, Rapp WD: Metabolically engineered soybean seed with enhanced threonine levels: biochemical characterization and seed-specific expression of lysine-insensitive variants of aspartate kinases from the enteric bacterium Xenorhabdus bovienii. Plant Biotechnol J 2011, 9(2):193-204.

59. Tabe LM, Droux M: Sulfur assimilation in developing lupin cotyledons could contribute significantly to the accumulation of organic sulfur reserves in the seed. Plant Physiol 2001, 126(1):176-187.

60. Gallardo K, Firnhaber C, Zuber H, Hericher D, Belghazi M, Henry C, Kuster H, Thompson R: A combined proteome and transcriptome analysis of developing Medicago truncatula seeds: evidence for metabolic specialization of maternal and filial tissues. Mol Cell Proteomics 2007, 6(12):2165-2179.

61. Noctor G, Mhamdi A, Chaouch S, Han Y, Neukermans J, Marquez-Garcia B, Queval G, Foyer $\mathrm{CH}$ : Glutathione in plants: an integrated overview. Plant Cell Environ 2012, 35(2):454-484.

62. Czechowski T, Stitt M, Altmann T, Udvardi MK, Scheible WR: Genome-wide identification and testing of superior reference genes for transcript normalization in Arabidopsis. Plant Physiol 2005, 139(1):5-17.

63. Sumner JB: The estimation of sugar in diabetic urine, using dinitrosalicylic acid. J Biol Chem 1924, 62:287-290.

64. Saeed Al, Sharov V, White J, Li J, Liang W, Bhagabati N, Braisted J, Klapa M, Currier T, Thiagarajan M, et al: TM4: a free, open-source system for microarray data management and analysis. Biotechniques 2003, 34(2):374-378.

doi:10.1186/1471-2229-13-206

Cite this article as: Matityahu et al.: Tobacco seeds expressing feedbackinsensitive cystathionine gamma-synthase exhibit elevated content of methionine and altered primary metabolic profile. BMC Plant Biology 2013 13:206

\section{Submit your next manuscript to BioMed Central and take full advantage of:}

- Convenient online submission

- Thorough peer review

- No space constraints or color figure charges

- Immediate publication on acceptance

- Inclusion in PubMed, CAS, Scopus and Google Scholar

- Research which is freely available for redistribution 